УДК 664.9.022

\title{
ПОДХОД К МАТЕМАТИЧЕСКОМУ ОПИСАНИЮ ПРОФИЛЕЙ НОЖЕЙ ДЛЯ РЫБОПЕРЕРАБАТЫВАЮЩЕГО ОБОРУДОВАНИЯ
}

\author{
О. В. Агеев
}

\section{THE APPROACH TO MATHEMATICAL DESCRIPTION OF THE PROFILES OF THE KNIVES FOR FISH PROCESSING EQUIPMENT}

\author{
O. V. Ageev
}

Показана необходимость разработки математического описания профилей ножей для резания рыбы. Проанализированы методы интерполяции траекторий рабочих органов в современном оборудовании с ЧПУ. С учетом технологии изготовления ножей для аналитического моделирования их профилей предлагается использовать полином третьего порядка. Разработана математическая модель, задающая форму передней криволинейной грани. Геометрия ножа характеризуется половинным ножом заточки, углом сопряжения граней, высотой фаски и половинной толщиной лезвия. При изменении параметров модели получены формы ножей с вогнутыми, выпуклыми, вогнуто-выпуклыми и выпукло-вогнутыми фасками. Модельная функция является непрерывной до второй производной включительно, за счет чего обеспечивается отсутствие на профиле уступов, изломов и скачков в кривизне. На участке от режущей кромки до боковой грани ножа функция имеет не более одной точки перегиба, что обеспечивает отсутствие местной волнистости на фаске. Определены условия монотонности на участке от острия ножа до точки перехода в боковую грань. Получено выражение для расчета координат точки перегиба указанной функции. Разработанное математическое описание профиля ножа является основой для постановки оптимизационных задач с целью определения наилучшей геометрии ножа с точки зрения ресурсосбережения. Наличие математической модели фаски позволяет определить оптимальный половинный угол заточки по критерию минимальной силы сопротивления резанию. Создана возможность рассчитать оптимальный половинный угол заточки, а также оптимальную половинную толщину ножа по критерию минимальной деформационной силы трения. Предложенный подход используется при постановке и решении вариационных задач по определению вида аналитических функций, задающих оптимальные профили режущих органов с минимальными вредными сопротивлениями.

рыба, резание, профиль, фаска, форма, нож, грань, модель

The paper shows the necessity of developing a mathematical description of the profiles of knives for cutting fish. The methods of interpolation of the working tools trajectories in modern equipment with CNC have been analyzed. Taking into account the technology of manufacturing knives for analytical modeling of their profiles, it is proposed to use a third-order polynomial. A mathematical model defining the shape of the 
front curvilinear edge has been developed. The knife geometry is characterized by a half angle, a mating angle of the edges, the height of the chamfer and half-thickness of the blade. By changing the model parameters, the shapes of knives with concave, convex, concave-convex and convex-concave chamfers have been obtained. The model function is continuous up to and including the second derivative, which ensures the absence of ledges, kinks, and jumps in the curvature on the profile. In the section from the cutting edge to the lateral edge of the knife, the function has no more than one inflection point, which ensures the absence of local waviness on the chamfer. The conditions of monotony in the section from the knife edge to the transition point to the lateral edge have been determined.

An expression for calculating the coordinates of the inflection point of the specified function has been obtained. The developed mathematical description of the knife profile is the basis for setting optimization problems in order to determine the best knife geometry from the point of view of resource conservation. The presence of a mathematical model of the chamfer allows you to determine the optimal half angle of grinding according to the criterion of the minimum force of resistance to cutting. The opportunity to calculate the optimal half angle of sharpening, as well as the optimal half thickness of the knife, according to the criterion of the minimum deformation friction force, has been created. The proposed approach is used in the formulation and solution of variational problems to determine the type of analytical functions that determine the optimal profiles of cutting tools with minimal harmful resistances.

fish, cutting, profile, chamfer, shape, knife, edge, model

\section{ВВЕДЕНИЕ}

В настоящее время широко применяется метод исследования оптимальных форм конструкций путем исследования функций, заданных полиномами, и ограничений на существующие классы решений. Численные данные, полученные по методу оптимизации с помощью полиномиальных функций, позволяют получать формы профилей, удовлетворяющих условию минимума сопротивления.

Геометрия ножей рыбоперерабатывающего оборудования определяется в процессе их проектирования. В дальнейшем информация по форме профиля используется при выполнении поверочных расчетов инструмента, а также в процессе конструкторско-технологической подготовки производства режущих органов. Внедрение в производство обрабатывающих центров с ЧПУ внесло существенные изменения в формы представления результатов проектирования изделий и привело к необходимости получения данных по аппроксимации формы ножа. Поскольку исходной информацией для работы программно-управляемого металлообрабатывающего оборудования являются результаты математического представления формируемой поверхности, необходимо рассматривать геометрию ножа на основе уравнений его профиля.

Исследованию геометрии режущих органов пищевого оборудования посвящен ряд работ в России и за рубежом. В работе [1] проанализировано влияние угла заточки ножа на процесс резания. В статье [2] исследован эффект внедрения лезвия в материал с учетом геометрии режущей кромки. Статьи $[3,4]$ посвящены определению оптимальных профилей рабочих органов для различных видов резания. В работах $[5,6]$ предложены подходы к численному определению сил со- 
противления при обработке вязкоупругих материалов. В исследовании [7] рассмотрен процесс скоростного резания и проанализированы его режимные параметры. В работе [8] проанализированы различные формы режущих инструментов и приведены рекомендации по их параметрам с точки зрения минимизации сил сопротивления. В статьях $[9,10]$ даны обзоры по моделированию разрезания вязкоупругих тканей с разработкой расчетных методик. В работе [11] предлагается подход к определению оптимального профиля горизонтального ножа для резания пищевого материала.

Вместе с тем, с учетом ценности существующих работ, в настоящее время отсутствует математическое описание формы рабочих органов рыбоперерабатывающего оборудования, необходимое для постановки и решения оптимизационных задач. Целью данной работы является разработка аналитического описания геометрии ножа, на основе которого представляется возможным определение сил сопротивления, а также деформационных сил трения для дальнейшей оптимизации профиля и обеспечения ресурсосбережения.

\section{МЕТОДЫ}

Профиль режущих органов в общем случае может иметь сложную форму и традиционно представлялся на чертеже в виде лекальных кривых и таблицами ординат группы точек. Однако математическое представление кривых профиля обладает рядом несомненных преимуществ. Оно весьма точно, и такие характеристики, как наклон и радиус кривизны, могут быть определены аналитически. Кроме того, математическое описание профиля может храниться в памяти ЭВМ и использоваться для автоматизированного проектирования рабочих органов и их изготовления на оборудовании с программным управлением. Требуется применение такого метода аналитического задания профиля ножа, который был бы применим на всех этапах: от эскизных проработок до технологической подготовки производства. Это позволяет моделировать форму режущего органа непосредственно в САПР, изменять геометрию граней заданным образом и обеспечивать изготовление изделия на автоматическом оборудовании.

В токарных и фрезерных станках наибольшее распространение получили позиционно-контурные системы управления, обеспечивающие как режим функционального управления, так и режим точного позиционирования. В то же время в эксплуатации находится оборудование с позиционными системами ЧПУ. Первой задачей программирования контурной обработки профиля является задание аналитического уравнения требуемой траектории движения исполнительного органа в плоских или пространственных координатах или значений координат семейства ее точек. Движения резца между опорными точками эквидистантной траектории задаются при помощи программно- реализованного интерполятора, на вход которого кадр за кадром подается информация в виде цифровых кодов, а на выходе формируется информация для каждой координаты исполнительного привода в виде последовательности импульсов (унитарного кода). Принципиально любая траектория рабочего органа при точении профиля ножа может быть аппроксимирована кривой линией, задаваемой в виде полинома Лагранжа.

Несмотря на то, что существует большое количество методов приближения кривых, которые, в принципе, возможно использовать для аппроксимации профиля ножа, наиболее универсальными свойствами обладают методы кусочно- 
гладкой аппроксимации сплайн-функциями. Наибольшее распространение в современных программно-управляемых станках получила интерполяция кривых кубическими сплайнами - функциями с непрерывной второй производной, являющихся на обрабатываемом участке кубическим полиномом. Такая кусочнокубическая интерполяция обладает практической ценностью в связи с тем, что позволяет на заданном множестве точек построить линию наименьшей кривизны. Вместе с тем, построение интерполятора, способного обеспечивать воспроизведение кривых 4-го, 5-го и более высоких порядков, связано с усложнением аппаратного и алгоритмического обеспечений системы ЧПУ, включающих перемножители, интеграторы, дифференциаторы, эстиматоры и другие вычислительные блоки.

Вышеизложенное приводит к тому, что при разработке математического описания профиля ножа в виде аналитического уравнения целесообразно использовать полином не выше третьего порядка. Это объясняется тем, что при выборе модели более высокого порядка, интерполятором станка с ЧПУ заданная форма профиля будет кусочно-гладко аппроксимирована кривыми третьего порядка (кубическими сплайнами). Использование аналитического описания геометрии грани в виде полинома третьего порядка позволяет избежать аппроксимации профиля кусочно-гладким сплайном, составленным из различных кубических полиномов, что упрощает вычисления и понижает численную неустойчивость, которая возрастает в случае аппроксимации кривых высоких порядков. В то же время кубический полином является кривой самой низкой степени, которая может иметь точки перегиба, что является достаточным для описания геометрии фасок и кромок ножей, применяемых для резания рыбы.

\section{МАТЕМАТИЧЕСКОЕ МОДЕЛИРОВАНИЕ}

Предъявим к математическому описанию профиля ножа следующие требования. Функция должна быть непрерывной до второй производной включительно, благодаря чему обеспечивается отсутствие на профиле уступов, изломов и скачков в кривизне. На участке от режущей кромки до боковой грани ножа функция должна иметь не более одной точки перегиба, что обеспечивает отсутствие местной волнистости на фаске. Также на данном участке функция должна быть положительной и монотонно возрастающей. Вторая производная функции должна быть положительной для тех участков, где фаска вогнута, и отрицательной для тех, где фаска выпуклая.

С учетом преимуществ использования рациональных многочленов при описании кривых линий применим рациональный многочлен 3-й степени, который запишем в виде:

$$
y(x)=\delta \cdot\left(b_{0}+b_{1} \cdot\left(x / h_{m}\right)+b_{2} \cdot\left(x / h_{m}\right)^{2}+b_{3} \cdot\left(x / h_{m}\right)^{3}\right) ; 0 \leq x \leq h_{m},
$$

где $h_{m}$ - высота передней криволинейной грани (фигурной фаски) ножа;

$\delta$ - половинная толщина ножа; $b_{0}, b_{1}, b_{2}, b_{3}$ - коэффициенты многочлена.

На рисунке показан общий вид симметричного профиля ножа с передними криволинейными наклонными гранями и прямым обухом. Введем следующие обозначения: $\alpha$ - половинный угол заточки ножа; $\theta$ - угол сопряжения передней наклонной грани с боковой гранью; $l$ - половинная толщина материала в состоянии равновесия. 
Поставим задачу описать функцией (1) монотонно возрастающие кривые, проходящие через точки $O(0,0)$ и $B\left(h_{m}, \delta\right)$. Через точку $O$ кривые должны проходить под заданным половинным углом заточки $\alpha$, через точку $B-$ под заданным углом сопряжения $\theta$. Указанные углы должны удовлетворять условиям: $0 \leq \alpha \leq 90^{\circ} ; 0 \leq \theta \leq 90^{\circ}$.

Из граничных условий на острие режущей кромки в точке $O$ :

$$
x=0 ; y(0)=0 ; y^{\prime}(0)=\operatorname{tg} \alpha .
$$

Из граничных условий в точке сопряжения граней $B$ :

$$
x=h_{m} ; y\left(h_{m}\right)=\delta ; y^{\prime}\left(h_{m}\right)=\operatorname{tg} \theta .
$$

Условие монотонности функции (1):

$$
x \in\left(0, h_{m}\right) ; y^{\prime}(x) \geq 0 .
$$

Определим первую и вторую производные функции (1):

$$
\begin{aligned}
& y^{\prime}(x)=\frac{\delta}{h_{m}^{3}} \cdot\left(b_{1} \cdot h_{m}^{2}+2 \cdot b_{2} \cdot h_{m} \cdot x+3 \cdot b_{3} \cdot x^{2}\right) ; \\
& y^{\prime \prime}(x)=\frac{2 \cdot \delta}{h_{m}^{3}} \cdot\left(b_{2} \cdot h_{m}+3 \cdot b_{3} \cdot x\right) .
\end{aligned}
$$

Из граничных условий (2), (3) имеем:

$$
\begin{gathered}
b_{0}=0 ; \quad b_{1}+b_{2}+b_{3}=1 ; \quad y^{\prime}(0)=\frac{\delta}{h_{m}} \cdot b_{1}=\operatorname{tg} \alpha \\
y^{\prime}\left(h_{m}\right)=\frac{\delta}{h_{m}} \cdot\left(b_{1}+2 \cdot b_{2}+3 \cdot b_{3}\right)=\operatorname{tg} \theta
\end{gathered}
$$

Из (7) получим систему уравнений, на основе которой определим коэффициенты многочлена (1). В результате выражения (1), (5), (6) примут следующий вид:

$$
\begin{aligned}
& y(x)= \delta \cdot\left(\frac{\operatorname{tg} \alpha}{\delta} \cdot x+\left(3-\frac{h_{m}}{\delta} \cdot(\operatorname{tg} \theta+2 \cdot \operatorname{tg} \alpha)\right) \cdot \frac{x^{2}}{h_{m}^{2}}+\left(\frac{h_{m}}{\delta} \cdot(\operatorname{tg} \theta+\operatorname{tg} \alpha)-2\right) \cdot \frac{x^{3}}{h_{m}^{3}}\right) ; \\
& y^{\prime}(x)= \operatorname{tg} \alpha+\frac{2}{h_{m}} \cdot\left(3 \cdot \frac{\delta}{h_{m}}-\operatorname{tg} \theta-2 \cdot \operatorname{tg} \alpha\right) \cdot x+\frac{3}{h_{m}^{2}} \cdot\left(\operatorname{tg} \theta+\operatorname{tg} \alpha-2 \cdot \frac{\delta}{h_{m}}\right) \cdot x^{2} ; \\
& y^{\prime \prime}(x)=\frac{2}{h_{m}} \cdot\left(3 \cdot \frac{\delta}{h_{m}}-\operatorname{tg} \theta-2 \cdot \operatorname{tg} \alpha+\frac{3}{h_{m}} \cdot\left(\operatorname{tg} \theta+\operatorname{tg} \alpha-2 \cdot \frac{\delta}{h_{m}}\right) \cdot x\right) .
\end{aligned}
$$

Определим условие монотонности функции (8). Указанная функция является монотонной, если ее первая производная (9) положительна: $y^{\prime}(x)>0$. Запишем следующее квадратное уравнение:

$$
y^{\prime}(x)=\operatorname{tg} \alpha+\frac{2}{h_{m}} \cdot\left(3 \cdot \frac{\delta}{h_{m}}-\operatorname{tg} \theta-2 \cdot \operatorname{tg} \alpha\right) \cdot x+\frac{3}{h_{m}^{2}} \cdot\left(\operatorname{tg} \theta+\operatorname{tg} \alpha-2 \cdot \frac{\delta}{h_{m}}\right) \cdot x^{2}=0 .
$$




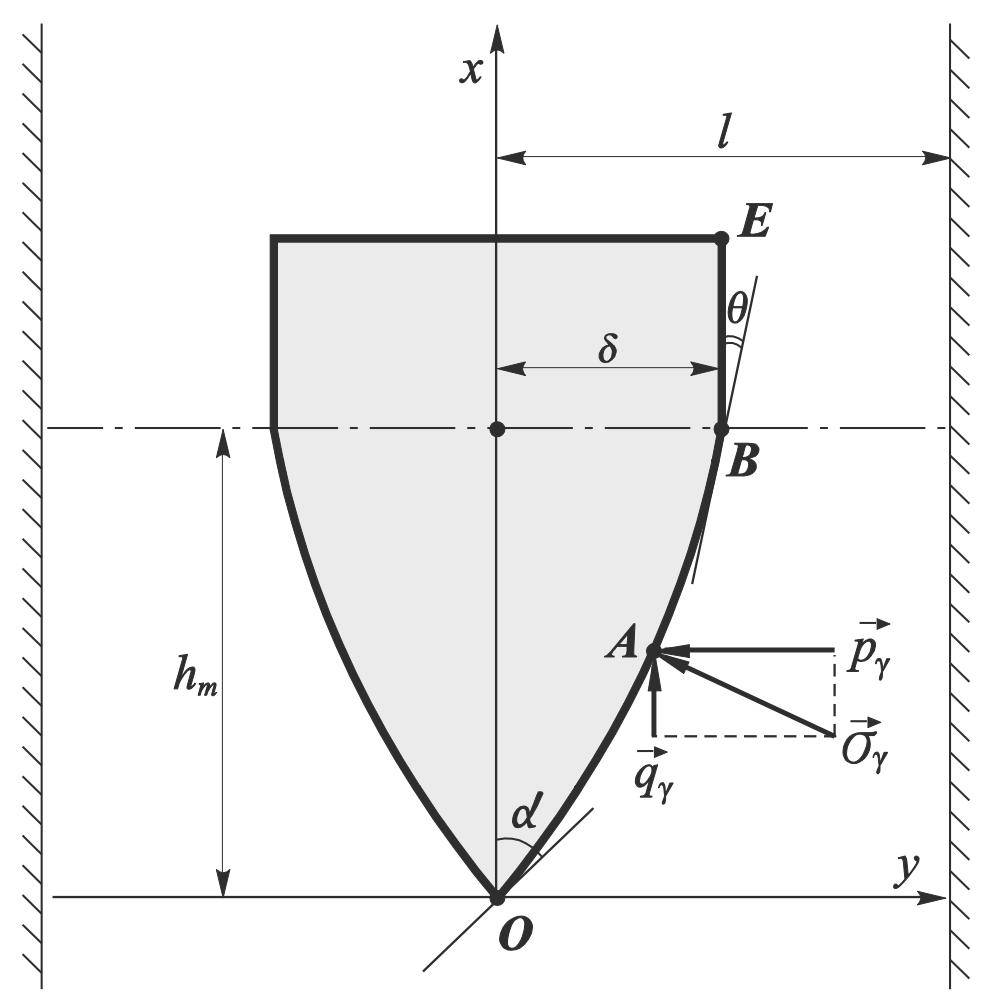

Рис. Вид криволинейного профиля ножа с прямым обухом Fig. View to a curved profile of a flat-back knife

Определим дискриминант уравнения (11):

$$
\begin{gathered}
D_{1}=\frac{4}{h_{m}^{2}} \cdot\left(3 \cdot \frac{\delta}{h_{m}}-\operatorname{tg} \theta-2 \cdot \operatorname{tg} \alpha\right)^{2}-\frac{12}{h_{m}^{2}} \cdot\left(\operatorname{tg} \theta+\operatorname{tg} \alpha-2 \cdot \frac{\delta}{h_{m}}\right) \cdot \operatorname{tg} \alpha= \\
=\frac{4}{h_{m}^{2}} \cdot\left[\left(3 \cdot \frac{\delta}{h_{m}}-(\operatorname{tg} \theta+2 \cdot \operatorname{tg} \alpha)\right)^{2}-3 \cdot\left(\operatorname{tg} \theta+\operatorname{tg} \alpha-2 \cdot \frac{\delta}{h_{m}}\right) \cdot \operatorname{tg} \alpha\right]= \\
=\frac{4}{h_{m}^{2}} \cdot\left[9 \cdot \frac{\delta^{2}}{h_{m}^{2}}+(\operatorname{tg} \theta+2 \cdot \operatorname{tg} \alpha)^{2}-6 \cdot \frac{\delta}{h_{m}} \cdot(\operatorname{tg} \theta+2 \cdot \operatorname{tg} \alpha)-3 \cdot \operatorname{tg} \theta \cdot \operatorname{tg} \alpha-3 \cdot \operatorname{tg}^{2} \alpha+\frac{6 \cdot \delta}{h_{m}} \cdot \operatorname{tg} \alpha\right]= \\
=\frac{4}{h_{m}^{2}} \cdot\left[\frac{9 \cdot \delta^{2}}{h_{m}^{2}}+\operatorname{tg}^{2} \theta+4 \cdot \operatorname{tg} \theta \cdot \operatorname{tg} \alpha+4 \cdot \operatorname{tg}^{2} \alpha-\frac{6 \cdot \delta}{h_{m}} \cdot \operatorname{tg} \theta-\right. \\
\left.-\frac{12 \cdot \delta}{h_{m}} \cdot \operatorname{tg}^{2} \alpha-3 \cdot \operatorname{tg} \theta \cdot \operatorname{tg} \alpha-3 \cdot \operatorname{tg}^{2} \alpha+\frac{6 \cdot \delta}{h_{m}} \cdot \operatorname{tg} \alpha\right]= \\
=\frac{4}{h_{m}^{2}} \cdot\left[\frac{9 \cdot \delta^{2}}{h_{m}^{2}}+\operatorname{tg}^{2} \theta+\operatorname{tg} \theta \cdot \operatorname{tg} \alpha+\operatorname{tg}^{2} \alpha-\frac{6 \cdot \delta}{h_{m}} \cdot \operatorname{tg} \theta-\frac{6 \cdot \delta}{h_{m}} \cdot \operatorname{tg} \alpha\right] .
\end{gathered}
$$

Функция (9) будет положительной, если дискриминант уравнения (11) меньше нуля: $D_{1}<0$. Определим, при каких значениях $\delta / h_{m}$ дискриминант $D_{1}$ будет меньше нуля. С учетом (12) запишем квадратное уравнение: 


$$
9 \cdot \frac{\delta^{2}}{h_{m}^{2}}-\frac{6 \cdot \delta}{h_{m}} \cdot(\operatorname{tg} \theta+\operatorname{tg} \alpha)+\operatorname{tg}^{2} \theta+\operatorname{tg} \theta \cdot \operatorname{tg} \alpha+\operatorname{tg}^{2} \alpha=0 .
$$

Найдем дискриминант уравнения (13):

$$
\begin{gathered}
D_{2}=36 \cdot(\operatorname{tg} \theta+\operatorname{tg} \alpha)^{2}-36 \cdot\left(\operatorname{tg}^{2} \theta+\operatorname{tg} \theta \cdot \operatorname{tg} \alpha+\operatorname{tg}^{2} \alpha\right)= \\
=36 \cdot\left(\operatorname{tg}^{2} \theta+2 \cdot \operatorname{tg} \theta \cdot \operatorname{tg} \alpha+\operatorname{tg}^{2} \alpha\right)-36 \cdot\left(\operatorname{tg}^{2} \theta+\operatorname{tg} \theta \cdot \operatorname{tg} \alpha+\operatorname{tg}^{2} \alpha\right)= \\
=36 \cdot\left(\operatorname{tg}^{2} \theta+2 \cdot \operatorname{tg} \theta \cdot \operatorname{tg} \alpha+\operatorname{tg}^{2} \alpha-\operatorname{tg}^{2} \theta-\operatorname{tg} \theta \cdot \operatorname{tg} \alpha-\operatorname{tg}^{2} \alpha\right)=36 \cdot \operatorname{tg} \theta \cdot \operatorname{tg} \alpha .
\end{gathered}
$$

Выражение (14) всегда положительно при $0 \leq \alpha \leq 90^{\circ} ; 0 \leq \theta \leq 90^{\circ}$, следовательно, уравнение (13) имеет два различных действительных корня:

$$
\frac{\delta}{h_{m}}=\frac{6 \cdot(\operatorname{tg} \theta+\operatorname{tg} \alpha)}{18} \pm 6 \cdot \sqrt{\operatorname{tg} \theta \cdot \operatorname{tg} \alpha}=\frac{1}{3} \cdot(\operatorname{tg} \theta+\operatorname{tg} \alpha \pm \sqrt{\operatorname{tg} \theta \cdot \operatorname{tg} \alpha}) .
$$

Таким образом, условие монотонности функции (8) при любых $x$ выглядит следующим образом:

$$
\frac{1}{3} \cdot(\operatorname{tg} \alpha+\operatorname{tg} \theta-\sqrt{\operatorname{tg} \alpha \cdot \operatorname{tg} \theta}) \leq \frac{\delta}{h_{m}} \leq \frac{1}{3} \cdot(\operatorname{tg} \alpha+\operatorname{tg} \theta+\sqrt{\operatorname{tg} \alpha \cdot \operatorname{tg} \theta}) .
$$

Условие монотонности функции (8) при $x \in\left(0 ; h_{m}\right)$ :

$$
\frac{\delta}{h_{m}} \geq \frac{1}{3} \cdot(\operatorname{tg} \alpha+\operatorname{tg} \theta-\sqrt{\operatorname{tg} \alpha \cdot \operatorname{tg} \theta}) .
$$

При выполнении условия (17) нарушение монотонности функции возможно только для $x$ вне отрезка $\left(0 ; h_{m}\right)$. Из (17) видим, что условие монотонности при заданном отношении половинной толщины ножа к высоте его передней наклонной грани зависит от половинного угла заточки и угла сопряжения.

Координату точки перегиба функции (8) определим из условия $y^{\prime \prime}(x)=0$ :

$$
\frac{2}{h_{m}} \cdot\left(3 \cdot \frac{\delta}{h_{m}}-\operatorname{tg} \theta-2 \cdot \operatorname{tg} \alpha+\frac{3}{h_{m}} \cdot\left(\operatorname{tg} \theta+\operatorname{tg} \alpha-2 \cdot \frac{\delta}{h_{m}}\right) \cdot x\right)=0
$$

откуда абсциссу точки перегиба получим в следующем виде:

$$
x_{p}=\frac{h_{m} \cdot\left(\operatorname{tg} \theta+2 \cdot \operatorname{tg} \alpha-3 \cdot \delta / h_{m}\right)}{3 \cdot\left(\operatorname{tg} \theta+\operatorname{tg} \alpha-2 \cdot \delta / h_{m}\right)} .
$$

Таким образом, располагая разработанной моделью (8), описывающей форму фаски ножа, в условиях стесненного сжатия материала представляется возможным рассчитать деформационные силы трения [12-14], а также силы сопротивления формы ножа $[15,16]$, используя дифференциальное уравнение состояния материала [17].

\section{ВЫВОДЫ}

1. Разработка математического описания профиля ножа создает теоретическую основу для постановки и решения оптимизационных задач по определению оптимальной геометрии режущего органа с целью обеспечения ресурсосбережения.

2. Наличие математической модели, описывающей форму фаски, позволяет определить оптимальный половинный угол заточки по критерию минимальной 
силы сопротивления резанию. Кроме того, существует возможность рассчитать оптимальный половинный угол заточки, а также половинную толщину ножа - по критерию минимальной деформационной силы трения.

3. Формализованное представление профиля в совокупности с математическими моделями для определения сил сопротивления формы ножа и деформационных сил трения позволяют выполнить постановку и решение вариационной задачи по определению вида функции, описывающей оптимальную форму профиля режущего органа с минимальным вредным сопротивлением.

\section{СПИСОК ИСПОЛЬЗОВАННЫХ ЛИТЕРАТУРНЫХ ИСТОЧНИКОВ}

1. Schuldt S, Arnold G, Kowalewski J, Schneider Y, Rohm H. Analysis of the sharpness of blades for food cutting. Journal of Food Engineering, 2016, no. 188, pp. $13-20$.

2. Spagnoli A, Brighenti R, Terzano M, Artoni F. Cutting resistance of soft materials: Effects of blade inclination and friction. Theoretical and Applied Fracture Mechanics, 2019, no. 101, pp. 200-206.

3. Atkins T. Prediction of sticking and sliding lengths on the rake faces of tools using cutting forces. International Journal of Mechanical Sciences, 2015, no. 91, pp. $33-45$.

4. Atkins T. Optimum blade configurations for the cutting of soft solids. Engineering Fracture Mechanics, 2006, no. 73, pp. 2523-2531.

5. Pagani M, Perego U. Explicit dynamics simulation of blade cutting of thin elastoplastic shells using «directional» cohesive elements in solid-shell finite element models. Computer methods in applied Mechanics and Engineering, 2015, no. 285, pp. 515-541.

6. Boisly M, Schuldt S, Kaestner M.G, Schneider Y, Rohm H. Experimental characterisation and numerical modelling of cutting processes in viscoelastic solids. Journal of Food Engineering, 2016, no. 191, pp. 1-9.

7. Schuldt S, Schneider Y, Rohm H. High-speed cutting of foods: Cutting behavior and initial cutting forces. Journal of Food Engineering, 2018, no. 230, pp. 55-62.

8. Han P, Che D, Pallav K, Ehmann K. Models of the cutting edge geometry of medical needles with applications to needle design. International Journal of Mechanical Sciences, 2012, no. 65, pp. 157-167.

9. Wang H, Chang L, Mai Y.-W, Ye L, Williams J.G. An experimental study of orthogonal cutting mechanisms for epoxies with two different crosslink densities. International Journal of Machine Tools and Manufacture, 2018, no. 124, pp. 117-125.

10. Takabi B, Tai B. L. A review of cutting mechanics and modeling techniques for biological materials. Medical Engineering \& Physics, 2017, no. 45, pp. 1-14.

11. Определение усилия со стороны ножа при резании с качением корнеклубнеплодов в измельчителе с горизонтальным вращающимся диском / П. А. Савиных [и др.] // Молочнохозяйственный вестник. - 2016. - № 3(23). -С. 62-75.

12. Ageev O, Naumov V, Fatykhov J. Mathematical modeling of the resistance force of the profile of a flat-back knife. Journal of Friction and Wear, 2019, vol. 40, no. 6, pp. 580-587. 
13. Агеев, О.В. Математическое моделирование силы сопротивления формы ножа с прямым обухом / О.В. Агеев, В.А. Наумов, Ю.А. Фатыхов // Трение и износ. -2019 . - Т. 40. - № 6. - С. 766-775.

14. Федоров, С.В. К исследованию совместимости трущихся поверхностей / С.В. Федоров // Вестник ВНИИЖТ. - 2016. - Т. 75. - № 5. - С. 283-288.

15. Агеев, О.В. Математическое моделирование деформационной силы трения ножа с различными параметрами шероховатости при резании рыбы / О.В. Агеев, В.А. Наумов, Ю.А. Фатыхов // Известия КГТУ. - 2019. - № 54. C. 77-90.

16. Расчет деформационной составляющей коэффициента трения шероховатой поверхности ножа при резании рыбы / О.В. Агеев [и др.] // Известия КГТУ. 2019. - № 55. - С. 177-189.

17. Агеев, О.В. Анализ соответствия реологических моделей структурномеханическим свойствам рыбы / О.В. Агеев [и др.] // Научный журнал СанктПетербургского национального исследовательского университета информационных технологий, механики и оптики. Серия: Процессы и аппараты пищевых производств. - 2018. - № 2(36). - С. 34-43.

\section{REFERENCES}

1. Schuldt S., Arnold G., Kowalewski J., Schneider Y., Rohm H. Analysis of the sharpness of blades for food cutting. Journal of Food Engineering, 2016, no. 188, pp. $13-20$.

2. Spagnoli A., Brighenti R., Terzano M., Artoni F. Cutting resistance of soft materials: Effects of blade inclination and friction. Theoretical and Applied Fracture Mechanics, 2019, no. 101, pp. 200-206.

3. Atkins T. Prediction of sticking and sliding lengths on the rake faces of tools using cutting forces. International Journal of Mechanical Sciences, 2015, no. 91, pp. $33-45$.

4. Atkins T. Optimum blade configurations for the cutting of soft solids. Engineering Fracture Mechanics, 2006, no. 73, pp. 2523-2531.

5. Pagani M., Perego U. Explicit dynamics simulation of blade cutting of thin elastoplastic shells using «directional» cohesive elements in solid-shell finite element models. Computer methods in applied Mechanics and Engineering, 2015, no. 285, pp. 515-541.

6. Boisly M., Schuldt S., Kaestner M. G, Schneider Y., Rohm H. Experimental characterisation and numerical modelling of cutting processes in viscoelastic solids. Journal of Food Engineering, 2016, no. 191, pp. 1-9.

7. Schuldt S., Schneider Y., Rohm H. High-speed cutting of foods: Cutting behavior and initial cutting forces. Journal of Food Engineering, 2018, no. 230, pp. 55-62.

8. Han P., Che D., Pallav K., Ehmann K. Models of the cutting edge geometry of medical needles with applications to needle design. International Journal of Mechanical Sciences, 2012, no. 65, pp. 157-167.

9. Wang H., Chang L., Mai Y.-W., Ye L., Williams J. G. An experimental study of orthogonal cutting mechanisms for epoxies with two different crosslink densities. International Journal of Machine Tools and Manufacture, 2018, no. 124, pp. 117-125. 
10. Takabi B., Tai B. L. A review of cutting mechanics and modeling techniques for biological materials. Medical Engineering \& Physics, 2017, no. 45, pp. 1-14.

11. Savinykh P. A., Aleshkin A. V., Bulatov S. J., Smirnov R. A. Opredelenie usiliya so storony nozha pri rezanii s kacheniem korneklubneplodov v izmel'chitele $\mathrm{s}$ gorizontal'nym vrashchayushchimsya diskom [Calculation of the knife force in cutting with a rolling blade in the chopper with a horizontal rotating disc]. Molochnokhozyaystvennyy vestnik, 2016, no. 3(23), pp. 62-75.

12. Ageev O. V., Naumov V. A., Fatykhov Yu. A. Mathematical modeling of the resistance force of the profile of a flat-back knife. Journal of Friction and Wear, 2019, vol. 40, no. 6, pp. 580-587.

13. Ageev O. V., Naumov V. A., Fatykhov Yu. A. Matematicheskoe modelirovanie sily soprotivleniya formy nozha s pryamym obukhom [Mathematical simulation of resistance force of a flat-back knife profile]. Trenie $i$ iznos, 2019, vol. 40, no. 6, pp. 766-775.

14. Fedorov S. V. K issledovaniyu sovmestimosti trushchikhsya poverkhnostey [On the study of the compatibility of rubbing surfaces]. Vestnik Vserossiyskogo nauchno-issledovatel'skogo instituta zheleznodorozhnogo transporta, 2016, vol. 75, no. 5, pp. 283-288.

15. Ageev O. V., Naumov V. A., Fatykhov Yu. A. Matematicheskoe modelirovanie deformatsionnoy sily treniya nozha s razlichnymi parametrami sherokhovatosti pri rezanii ryby [Mathematical simulation of deformation friction force of knife with different parameters of roughness during fish cutting]. Izvestiya Kaliningradskogo gosudarstvennogo tekhnicheskogo universiteta, 2019, no. 54, pp. 77-90.

16. Ageev O. V., Naumov V. A., Fatykhov Yu. A., Samoilova N. V. Raschet deformatsionnoy sostavlyayushchey koeffitsienta treniya sherokhovatoy poverkhnosti nozha pri rezanii ryby [Calculation of deformation friction coefficient of rough knife surface during fish cutting]. Izvestiya Kaliningradskogo gosudarstvennogo tekhnicheskogo universiteta, 2019, no. 55, pp. 177-189.

17. Ageev O. V., Naumov V. A., Fatykhov Yu. A., Samoilova N. V. Analiz sootvetstviya reologicheskih modeley strukturno-mekhanicheskim svoystvam ryby [Correspondence analysis of rheological models to the structural-mechanical properties of fish]. Nauchnyy zhurnal Sankt-Peterburgskogo natsional'nogo issledovatel'skogo universiteta informatsionnykh tekhnologiy, mekhaniki i optiki. Seriya: Processy i apparaty pishchevykh proizvodstv, 2018, no. 2(36), pp. 34-43.

\section{ИНФОРМАЦИЯ ОБ АВТОРЕ}

Агеев Олег Вячеславович - Калининградский государственный технический университет; кандидат технических наук; доцент кафедры пищевых и холодильных машин; E-mail: oleg.ageev@klgtu.ru

Ageev Oleg Viatcheslavovich - Kaliningrad State Technical University; PhD in Engineering, Associate Professor, Department of Food and Refrigeration Machines; E-mail: oleg.ageev@klgtu.ru 